\title{
SCOPING REVIEW
}

\section{Heather Leduc-Pessah, PhD ${ }^{1,2}$ \\ Samuel K. Jensen, PhD ${ }^{1,2}$ \\ Christopher Newell, PhD ${ }^{1,2}$}

${ }^{1}$ Faculty of Medicine, Cumming School of Medicine, University of Calgary, Calgary, Alberta, Canada

2 All authors equally contributed to this work

\section{An overview of the adverse effects of cannabis use for Canadian physicians}

\begin{abstract}
Purpose: Cannabis is the most widely used illicit substance and one of the most commonly used psychoactive substances in the world, preceded only by alcohol, tobacco and caffeine. Recent changes in legislation regarding cannabis use in Canada and potential upcoming changes worldwide may have a further impact on the prevalence of cannabis use. Thus, it is critical to understand the risks and potential adverse health effects of acute and long-term cannabis use. Current literature is lacking in many areas surrounding cannabis use, and for the most part is unable to provide clear associations once confounding variables are considered. Here we provide a general overview of the history of cannabis, the physical and mental health consequences, and the risks to specific groups.
\end{abstract}

Source: A scoping search of published articles in PubMed from the start date (1946) until 2018.

Principal finding: Current evidence supports an association between cannabis use and mild respiratory and cardiac effects, but no clear increased risk of cancer. Psychiatric disorders, including schizophrenia and anxiety, show associations with cannabis use; however, a causal effect of cannabis use is unclear. While no evidence for increased risk in pregnancy has been found, risk is still undetermined. Youth may be at a greater risk as earlier initiation of use increases the risk of adverse health effects.

Conclusion: Overall, evidence for direct and long-term adverse effects of cannabis use is minimal and additional longitudinal studies will be required to better delineate unidentified effects.

Correspondence to:

Christopher Newell

Email: cnewell@ucalgary.ca 
Recorded cannabis use dates to as early as 2,000 B.C. Throughout this time period, cannabis was used for its medicinal benefits as well as for ceremonial and ritual practices. Medically, cannabis has been used for the treatment of various ailments, including rheumatism, pain and malaria, and as an anesthetic. In the 1900s, fear surrounding the abuse of other drugs, such as opium and cocaine, led to the criminalization of cannabis in numerous countries around the world, despite minimal evidence to support its potential health consequences and addiction liability. Since this time, studies on the potential health benefits and adverse effects of cannabis have been and continue to be essential to better understand the appropriate indications for cannabis.

In recent years, significant political reforms regarding the use of medicinal and recreational cannabis may lead to an increase in the use of cannabis globally. As a result, it is critical to understand the potential long-term effects of cannabis use and risks of specific health concerns. In this literature review we outline a history of cannabis use and the negative effects associated with it that led to its widespread criminalization. We then describe the active components of cannabis and review the current literature investigating the physical and mental health consequences of cannabis use.

Some of the major adverse effects thought to be associated with cannabis use include the risk of lung cancer associated with smoking cannabis, the development of cardiovascular disease, and a potential impact on the developing nervous system in youth. Cannabis use is also thought to be associated with a variety of mental health disorders including acute or chronic psychosis, anxiety, and schizophrenia, among others. The addiction potential of cannabis has been heavily debated and we provide an overview of the evidence supporting or refuting this claim. In addition, we explore the impact of cannabis on specific populations including pregnant women, youth and those with previous mental health concerns and discuss any evidence for contraindications to cannabis use. Collectively, this literature review will provide an overview of the adverse effects associated with cannabis use, an essential consideration as its medicinal and recreational use becomes more prevalent around the world. This information is intended to inform clinical practitioners on how to advise patients regarding the risks associated with both medicinal and recreational cannabis.

\section{Sources}

We conducted a scoping search of published articles in PubMed from the start date (1946) until 2018. We included all published articles and study designs including case reports, case series, cohort studies, case-control studies, clinical trials, basic science articles and review articles. Studies were excluded if they were not written in English. No restriction was imposed on sex, race, population, or geographical location of study. Initial search for relevant content included terms "cannabis" or "health consequences" or "adverse effects" in the title and/or abstract. Further comprehensive searches were conducted to each specific health consequence discussed in the review. We summarized the results using a descriptive (noninterpretive) narrative synthesis and content analysis. Bibliographic management was done using Mendeley software to collect and analyze the references. Results are summarized in Figure 1 and Table 1.

\section{Historical context}

Cannabis sativa, Cannabis indica and, to a lesser extent, Cannabis ruderalis (known colloquially as marijuana, weed, etc.), have been utilized for cultural and spiritual purposes for over 5,000 years [1]. Interestingly, medicinal utilization of cannabis has only been documented once since 400 A.D. by carbon dating of ashes that identified the psychoactive compound, THC [2]. Composed of approximately 750 chemicals, including 104 cannabinoids, cannabis is the most widely consumed psychoactive substance worldwide. Data generated in 2013 estimated that 180 million people ( $3 \%$ of the world's population) consume cannabis annually (compared with $2 \%$ for cocaine and $0.2 \%$ for opiates) [3].

In Canada, cannabis has been prohibited since it was added to the Confidential Restricted List in 1923 under the Narcotics Drug Act Amendment Bill [4]. The addition of cannabis to the list of prohibited drugs in Canada followed a wave of legislation banning psychoactive substances, beginning with the Opium Act of 1908 [5]. Amidst almost a century of illegality, cannabis remains the most widely used illicit drug in Canada, with a 2012 report indicating that $42.5 \%$ of the population reported having ever used cannabis, and $12.2 \%$ reported use in the past year [6]. Although recreation use was deemed illegal, the Supreme Court of Canada ruled in favour of legislation providing access to cannabis for medical purposes on July 30th, 2001. Under this ruling, Health Canada developed two categories of patients who are eligible for access to medical cannabis. Category 1 encompasses end-of-life care and includes patients presenting with a series of symptoms including 1) seizures from epilepsy, 2) severe pain from multiple sclerosis or spinal cord damage, 3) severe pain from arthritis or 4) severe pain from cachexia, anorexia, weight loss and/or severe nausea from cancer or HIV/AIDS infections. Category 2 includes patients presenting with debilitating symptoms from conditions not explicitly included in Category 1, and each 


\section{Cognition}

- Acute - Impairment in cognition, possible euphoria or dysphoria.

- Evidence for ongoing cognitive impairment with heavy, frequent use.

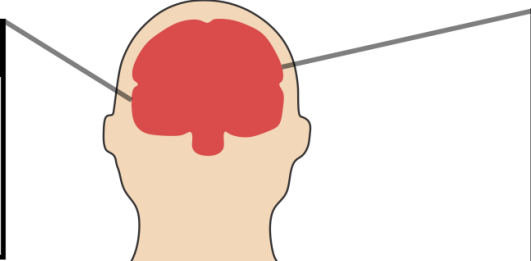

\section{Respiratory}

- Chronic bronchitis.

- COPD exacerbations if pre-existing.

- No deficits in pulmonary function tests acutely, long-term lung function deteriorates earlier.

\section{Lung Cancer}

- No identified independent association between smoking cannabis and lung cancer.

\section{Cancer (others)}

- No strong association with any cancers known to be associated with tobacco smoking.

- Possible increased risk of testicular cancer with heavy cannabis use.

\section{Youth}

- Some evidence for adverse impact on brain development.

- Earlier initiation of use increases risk of mental health disorders.
Mental Health

- Cannabis-induced psychosis can rarely occur.

- Schizophrenia association with cannabis use.

- Increased risk of anxiety.

- Depression unclear association.

- Overall negative impact on individuals with pre-existing mental health concerns.

\section{Cardiac}

- No long term changes in cardiovascular mortality.

- Acute - increased risk of MI.

- Acute - tacchycardia and hypotension.

- Chronic - bradycardia and normotension.

\section{Pregnancy}

- Unable to identify direct harm in current studies.

- Not investigated for safety in pregnancy.

\section{Gastrointestinal}

- Cannabinoid hyperemesis syndrome with heavy use.

FIGURE 1. Summary of the potential adverse health consequences of cannabis use 
application for medicinal cannabis must be endorsed by a medical practitioner.

Amidst growing public acceptance of recreational cannabis use and the logistical inability of the existing control systems in Canada, legalization of cannabis was proposed by the Liberal Party of Canada in 2012. Passed in 2017 and effective October 17th, 2018, the Cannabis Act (Bill C-45) legalized the recreational use of cannabis in Canada. While many other countries across the world retain the illegality of cannabis [7], the issue surrounding legalizing this illicit drug has received considerable public and political attention in recent years, culminating in the decision to amend the 1923 bill. However, there remains a paucity of research investigating the short- and long-term impact of cannabis on the physical and mental health of consumers.

\section{Psychoactive compounds found in cannabis}

Tetrahydrocannabinol (THC) is the primary psychoactive compound found in cannabis and is the most widely studied component. However, cannabis contains at least 65 other cannabinoids, the effects of which have received minimal investigation [8]. The other major component of interest is cannabidiol (CBD), which produces many of the same effects as THC but with comparatively minimal intoxication.

Cannabinoids act on specific receptors found within the central nervous and peripheral organ systems [9]. These receptors are part of a naturally occurring endocannabinoid system where intrinsically produced cannabinoids (primarily anandamide and 2-arachidonoylglycerol) regulate the activity of other neurotransmitters to modulate cognition, emotion and memory. Two types of cannabinoid receptors have been identified, CB-1 and CB-2 receptors, which differ based on their location of expression. The CB-1 receptor is primarily found within the central nervous system and regulates the psychoactive and cognitive effects of cannabinoids, whereas CB-2 is primarily found on peripheral cells and is involved in the regulation of organ and immune system function.

While significant effort has been made to define the pharmacological effects of cannabinoid and their individual receptors, particularly in animal models, a limited amount of this information has been translated into humans. Cannabis contains cannabinoids that activate both receptors; thus, the public consumption of cannabis is likely to cause effects that are mediated by the entire endocannabinoid system, rather than specific aspects of it. Of interest, cannabis strains that contain primarily CBD, with limited to no THC, have been developed to produce the beneficial effects of activating the endocannabinoid system without the associated intoxication. However, limited study on these strains and their efficacy at achieving this has been done. Thus, the rest of this article will not make a distinction between different strains or cannabinoids and will simply refer to cannabis.

\section{Physical health concerns}

\section{Impact on respiratory system}

Chronic obstructive pulmonary disease (COPD) is a progressive narrowing of respiratory airways that can lead to death. The primary presenting symptoms are shortness of breath, a productive cough (sputum) and wheezing [10]. Tobacco smoking is the most common cause of COPD, which includes emphysema, chronic bronchitis and refractory asthma. Currently, 10 cross-sectional or prospective cohort studies have investigated the impact of smoking cannabis on the development of COPD symptoms [11]. An issue in comparing cannabis smokers to controls is that cannabis smokers also commonly consume tobacco. However, after adjusting for tobacco, cannabis smokers have demonstrated significantly more COPD symptoms than healthy controls [12]. Interestingly, cannabis smokers are more likely to report bouts of wheezing and a productive cough, although no change in shortness of breath compared to controls [13].

Histological analysis of bronchoscopy specimens has been performed to provide insight as to the underlying mechanism behind cannabis and chronic bronchitis. Fligiel et al. [14] compared bronchial mucosa biopsies from individuals classified as never smokers, cannabis-only smokers, tobacco-only smokers or cannabis-and-tobacco smokers. This study revealed that tobacco-only smokers and cannabis-only smokers demonstrated similar histological changes to their bronchial mucosa. This was characterized by marked destruction of the ciliated columnar epithelium lining the bronchi, and their replacement by mucus-secreting goblet cells. The productive cough identified as a symptom of cannabis consumption may therefore be a result of this histopathological shift to mucus-secreting goblet cells. Roth et al. [15] further developed this theory in a follow-up study involving endoscopy of a similarly divided patient population. This work identified evidence of bronchial inflammation, injury of the mucosa and increased secretions in cannabis-only smokers that was comparable to the bronchi of tobacco-only smokers.

Although smoking cannabis has been associated with some of the symptoms of COPD (productive cough and wheezing), there has been no evidence of increased risk of COPD in cannabis-only smokers [12]. Four longitudinal studies have been completed investigating the impact of smoking cannabis on lung function over time. Of these studies, three identified no impairment in lung function, 
although cannabis-only smokers do lose lung function more quickly than non-smokers $[12,16,17]$. The fourth study, by Sherrill et al. [18], examined non-tobacco cigarette smoking, as a representation of cannabis smokers, and found that FEV1 was significantly decreased compared with nonsmoking controls. However, FEV1 in non-smokers was found to increase over time, although FEV1 has been previously shown to decline with age. Interestingly, research has found that cannabis-only smokers have increased forced vital capacity compared with controls as measured by spirometry, which may be associated with deep inhalations common in smoking cannabis [19]. On another note, several studies have compared whole body plethysmography between non-smokers and cannabis smokers. Each of these studies found specific airway conductance to be decreased in cannabis smokers [20-23]. These studies provide evidence to support the findings of Roth et al. [15], where the primary and secondary bronchi were shown to be visually impaired (inflamed and edematous). However, in these plethysmography studies, the measure of how much oxygen travels from the alveoli of the lungs to the bloodstream was not elevated in cannabis smokers. As a measure of alveolar function, these demonstrate that cannabis smoking indeed impacts the large airways but may not lead to emphysema. The link between cannabis smoking and emphysema has been further evaluated using high-resolution computed tomography scanning in two studies. Aldington et al. [21] identified no evidence of COPD, whereas Morris et al. [24] identified that cannabis-only smokers were found to have significantly less COPD than controls.

In response to the inhalation of tobacco smoke, the number of alveolar macrophages and activated macrophages has been found to increase [25]. Bronchoalveolar lavage research has demonstrated that smoking cannabis also leads to an increase in the number of alveolar macrophages [26]. Interestingly, ex vivo functional research has demonstrated that alveolar macrophages from cannabis-only smokers have impairments in their cytokine mediated activities [27]. Thought to be linked to the immunosuppressive actions of THC, these changes could both lead to increased susceptibility to infectious lung diseases and protection from inflammatory changes leading to COPD.

Smoking cannabis may be linked to an increased risk for contracting lower respiratory tract infections [11]. Firstly, the histopathological shift from ciliated columnar epithelium to mucus-secreting goblet cells may provide a substrate for pathogenic bacteria to colonize within the lungs. Furthermore, the shift from ciliated columnar epithelium may inhibit the transit of mucus from the airways resulting in stasis of mucus potentially colonized by pathogenic bacteria. Secondly, the immunosuppressive effects of THC on alveolar macrophages may inhibit recognition of inhaled pathogens and activation of phagocytic cells [28]. This may lead to compromised lung function against pathogenic bacteria. Thirdly, cannabis has been frequently contaminated with pathogenic gram-negative bacteria the fungus Aspergillus fumigatus. This fungus is the most common cause of invasive fungal infection in immunocompromised hosts and can be easily acquired through smoking contaminated cannabis [29].

\section{Impact on gastrointestinal system}

Cannabis is considered one of the oldest pharmacological therapies for nausea and vomiting, dating back hundreds, if not thousands, of years [30]. Recent research comparing the effects of cannabis to modern anti-emetic medicines has even demonstrated that cannabis may be better suited to alleviate nausea [31]. Paradoxically, there is evidence to suggest that chronic cannabis use can also result in excessive bouts of nausea and vomiting. Cannabinoid hyperemesis syndrome (CHS) was first characterized by Allen et al. [32] in nine Australian patients and is characterized by chronic cannabis use, cyclic episodes of nausea and vomiting and the learned behavior of frequent hot bathing [33]. Unique from cyclic vomiting syndrome, which is a mitochondrial disorder [34], CHS is classified as a recurrent disorder divided into three phases: pre-emetic; hyperemetic; and recovery. Patients commonly present themselves to the emergency room during the hyperemetic phase and require intravenous antiemetic medicines and fluids, although the only proven therapy is cessation of cannabis use [35]. While the mechanism of action has yet to be elucidated, it has been hypothesized that CHS may be linked to chronic cannabis consumption and to the long half-life of certain THC components, which result in eventual toxic accumulation [31].

\section{Impact on cardiovascular system}

As previously described, the endocannabinoid system is composed of the cannabinoid receptors (CB-1 and CB-2) and the endogenous counterparts of THC [36]. Both cannabinoid receptors have been identified in a number of tissues including liver, muscle, fat and brain, whereas CB-2 receptors are expressed in the spleen and immune cells [37]. The CB-1 receptor agonism in mice has been shown to cause prolonged hypotension [38], while CB-2 receptors present on cardiomyocytes and coronary endothelium may function to regulate infiltration of immune cells [39]. Through control of inflammation of cardiac cells, the agonism of CB-2 may therefore be cardioprotective. 
Contrarily, a reliable marker of cannabis consumption is tachycardia [40]. The mechanism behind this phenomenon is thought to be a result of cannabis-induced vasodilation leading to reflex tachycardia. Interestingly, tolerance to cannabis induced tachycardia has been characterized only two weeks after prolonged use and chronic cannabis smokers have been found to have decreased heart rate and increased blood volume and to lack bouts of hypotension [41]. Although these results suggest acute effects of cannabis on heart rate, several case reports have also been published identifying smoking cannabis as a trigger for atrial fibrillation and fatal ventricular tachycardia [42], [43]. Further investigation of these cardiovascular phenomena requires controlled research trials to identify any causation between smoking cannabis and sudden cardiac death.

In recent years, case studies have characterized ischemic cardiac events in cannabis smokers as well as cannabistriggered myocardial infarctions in young healthy male patients $[43,44]$. Although these individual reports should also be more dutifully investigated to conclude any risk associated with smoking cannabis and cardiac ischemia, there is concern that the patients being impacted are young, otherwise healthy, males. Apart from the age and lack of comorbidities, these cardiac events are also commonly triggered events during or recently after bouts of strenuous physical activity. This is concerning since these patients do not represent the characteristic demographics of cardiac patients. In order to elucidate the potential risk of smoking cannabis on acute myocardial infarctions (MI), Mittleman et al. [45] interviewed patients previously diagnosed with an MI to compare self-reported use of cannabis at the time of symptoms. Smoking cannabis was found to be a trigger for MI, with risk for MI 4.8 times greater than baseline in the hour following cannabis use. However, research by Frost et al. [46], using the same cohort of patients, was unable to demonstrate a statistical association between smoking cannabis and mortality after 18 years of follow-up. These results suggest that although cannabis use may result in an increased risk for acute cardiovascular events, no differences in long-term cardiovascular mortality have yet been proven.

There have also been reports of cannabis use precipitating ischemic or transient cerebrovascular events. In 2013 there were approximately 60 cases reports in the literature, with this number increasing to 100 by 2015 [47]. Again, being predominantly reported in young, otherwise healthy, male patients, the proposed mechanism behind these cerebrovascular events implicates vasospasm or reversible cerebral vasoconstriction [48]. This is supported by the reversal of cerebral vascular changes as documented by vascular imaging in all patients who stopped consuming cannabis [48]. However, the cause and effect between cannabis and cerebrovascular events has yet to be established and the number of cannabis associated cerebrovascular events is extremely infrequent in relation to the widespread use of cannabis [49].

\section{Impact on cancer development}

Tetrahydrocannabinol and the associated cannabinoids are not carcinogenic, as demonstrated by testing in bacteria and animal models [50]. However, cannabis cigarettes are known to contain procarcinogenic components, such as benzpyrene, a human carcinogen found in tobacco smoke [51]. It is, therefore, logical to assume that smoking cannabis may result in similar cancers as smoking of tobacco, namely lung and upper respiratory tract. The existing literature examining lung cancer risk and cannabis consumption has been mixed. Histological reports have noted that habitual cannabis smokers have similar squamous epithelial abnormalities (cellular dysplasia and squamous metaplasia) as noted in tobacco smokers [14]. Elevated levels of oncogenes and oncoproteins, evidenced by immunohistochemical testing, has also been found in bronchial biopsies from cannabis smokers [52]. A large Swedish conscription study (49,343 individuals) examined the association between smoking cannabis more than 50 times before $18-20$ years of age and lifetime lung cancer risk over the subsequent 40 years [53]. Although this study concluded that cannabis smoking was linked to lung cancer development, there was no adjustment for lifetime tobacco use, a vital confounding variable. An epidemiological African study also identified cannabis smoking as a risk factor for lung cancer although the study cohort was also smoking tobacco [54]. Furthermore, a recent study pooling the results of several case-control studies found that there was little evidence for an increased risk of lung cancer in habitual or long-term cannabis smokers [55].

Collectively, these data demonstrate that there is no identified independent link between smoking cannabis and lung cancer. In an effort to explain the potential mechanism for these effects, researchers have investigated the role of THC and associated cannabinoids on tumorigenesis. Many researchers have described a tumour suppressive effect of both THC and other cannabinoids on lung cancer and other malignant neoplasms [56]. It has been hypothesized that the procarcinogenic components of smoking cannabis may be counteracted by the anti-proliferative, anti-angiogenic effects that THC and associated cannabinoids have on various malignancies [56]. Finally, a large (64,855 individuals) Californian retrospective cohort study, completed by Sidney et al. [57], found no association between smoking cannabis and six cancers - colorectal, lung, melanoma, prostate, breast 
and cervix. These results were adjusted for socioeconomic status (SES), alcohol consumption and cigarette smoking, concluding that young patients who smoke cannabis are at no greater risk than non-users. Going forwards, more and better designed in-depth epidemiological and populationbased research is required to control for the effects of tobacco smoking in order to clarify the impact of cannabis smoking on cancer risk.

The correlation between the risk of developing testicular cancer and cannabis use has also been explored. A systematic review and meta-analysis by Gurney et al. examined the role of cannabis use and development of testicular germ cell tumors (TGCT)[58]. Interestingly, there was no association between the development of TGCT and ever-use of cannabis; however, there was an increase of TGCT development with weekly (or greater) cannabis use, prolonged use ( $>10$ years vs. never use) and current use. While interesting, it must be noted that these comparisons were made using data from only three studies, which contained data primarily collected from the United States of America in the 1990s. Similar examination of testicular cancer development and cannabis use was also explored in the same large Swedish conscription study (49,343 individuals) as mentioned above [59]. These data also demonstrated that there was no association between lifetime ever-use of cannabis and the development of testicular cancer. Although, there was an association with heavy cannabis use (smoking cannabis more than 50 times before the 18-20 years of age) and development of testicular cancer over the subsequent 40 years. Collectively, these data demonstrated that duration and frequency of cannabis use may impact the development of testicular cancer.

\section{Cognitive and mental health concerns}

In addition to the potential physical health consequences, considerable evidence shows an increased risk of mental health disorders associated with cannabis use [60]. However, there is insufficient evidence to fully describe these associations. The high degree of overlap between the risk factors for initiation of cannabis use and the adverse outcomes associated with repeated cannabis use makes it difficult to differentiate pre-existing risks from direct consequences of cannabis use. Thus, proving cannabis has a causal effect on any of these disorders is extremely difficult. Several lines of evidence show that pre-existing or underlying mental health disorders are associated with an increased likelihood for initiation of cannabis use, suggesting possible reverse causation. It is important to consider these factors when interpreting the association studies, and to consider whether these studies properly control for pre- existing conditions and exclude confounding variables. In this section, the acute and long-term effects of cannabis use on cognition, psychosis, schizophrenia, anxiety and depression are described. In each association, an earlier age of onset of cannabis use is predictive of severity or increased risk of mental health consequences and psychiatric disorders. One hypothesis for this association is the impact of cannabis on the developing brain when used at younger ages [61], which is described in more detail in subsequent sections of this review.

\section{Cannabis use in individuals with pre-existing mental health conditions}

Concern exists for cannabis use both for the development of mental health conditions in predisposing individuals and for possible negative effects of cannabis use on individuals with existing mental health disorders. Indeed, cannabis use appears to increase the severity of psychotic disorders such as schizophrenia [62]. Furthermore, people with bipolar disorder experience more manic episodes when compared with non-users; however, cannabis use does not appear to have a substantial effect on the severity or frequency of depressive episodes [63]. Overall, current evidence suggests cannabis use should be avoided or at least minimized in individuals with pre-existing mental health concerns.

\section{Impact of acute consumption}

The short-term effects of cannabis use are dependent on the route of administration, amount used, prior exposure to cannabis as well as any concurrent drug use. The onset, duration and peak of cannabis effects are also variable and dependent on these factors. Inhalation of cannabis smoke typically produces psychoactive effects within 15 to 30 minutes, whereas onset of effect after ingestion varies from 30 minutes to three hours and the overall duration of these effects can range from two to 12 hours [64]. The most commonly experienced psychoactive effects of acute cannabis exposure include euphoric, relaxing effects primarily mediated by the binding of the active components at endogenous cannabinoid receptors in the brain. In a subset of users, cannabis may have acute dysphoric or psychotic effects, including mild to severe anxiety, panic, delusions, paranoia, auditory hallucinations and other changes in perceptions [65]. The most severe presentations of acute cannabis intoxication are associated with cannabisinduced psychosis (CIP), which can be serious and may require treatment. Cannabis-induced psychosis following cannabis exposure can be temporary, leading to complete remission; but in some individuals, CIP leads to long-term 
effects and is associated with an increased risk for developing

psychiatric disorders later in life [66].

\section{TABLE 1. Summary of the health consequences of cannabis use based on current scientific evidence}

\begin{tabular}{|c|c|c|}
\hline $\begin{array}{l}\text { Altered mental } \\
\text { status }\end{array}$ & $\begin{array}{l}\text { Short-term effects of exposure to cannabis: euphoric, dysphoric, psychotic effects, anxiety, panic, } \\
\text { delusions, paranoia, auditory hallucinations and changes in perception. }\end{array}$ & Ashton, 2001 \\
\hline Cognition & $\begin{array}{l}\text { Acutely cannabis alters mental status, memory and cognition. } \\
\text { Prolonged cognitive impairment can occur following heavy, frequent use with no return to } \\
\text { baseline between cannabis use. }\end{array}$ & $\begin{array}{l}\text { Nugent et al, } 2017 \\
\text { Lenné et al, } 2010 \\
\text { Hindocha et al, } 2017 \\
\text { WHO, } 2016 \\
\text { Meier, 2012, Auer, } 2016\end{array}$ \\
\hline Anxiety & Increased risk of anxiety in cannabis users. & Twomey, 2017 \\
\hline $\begin{array}{l}\text { Cannabis- } \\
\text { induced } \\
\text { psychosis }\end{array}$ & Cannabis-induced psychosis is a rare adverse effect 24 hours to seven days after use. & $\begin{array}{l}\text { Shah et al, } 2017 \\
\text { Shrivastava et al, } 2011\end{array}$ \\
\hline Addiction & Risk for the development of substance (cannabis) use disorder. & $\begin{array}{l}\text { Budney et al, } 2007 \\
\text { Copeland et al, } 2009\end{array}$ \\
\hline $\begin{array}{l}\text { Pre-existing } \\
\text { mental health } \\
\text { concerns }\end{array}$ & $\begin{array}{l}\text { Cannabis has a negative impact, increasing the symptoms and frequency of episodes in } \\
\text { individuals with pre-existing psychiatric conditions, including schizophrenia. }\end{array}$ & $\begin{array}{l}\text { Foti et al, } 2010 \\
\text { Schoeler et al, } 2016 \\
\text { Zammit et al, } 2008 \\
\text { Buckner et al, } 2012\end{array}$ \\
\hline $\begin{array}{l}\text { Chronic } \\
\text { bronchitis }\end{array}$ & Cannabis use can lead to development of chronic recurrent bronchitis. & Fligiel et al, 1997 \\
\hline $\begin{array}{l}\text { COPD } \\
\text { exacerbations }\end{array}$ & Exacerbation of pre-existing COPD with cannabis use. & $\begin{array}{l}\text { Hancox et al, } 2015 \\
\text { Tan et al, } 2009\end{array}$ \\
\hline $\begin{array}{l}\text { Hyperemesis } \\
\text { syndrome }\end{array}$ & Cannabinoid-induced hyperemesis syndrome can occur with heavy use. & $\begin{array}{l}\text { Allen et al, } 2004 \\
\text { Goyal et al, } 2017\end{array}$ \\
\hline $\begin{array}{l}\text { Myocardial } \\
\text { infarction }\end{array}$ & Increased risk of myocardial infarction in the one hour following cannabis use. & Mittleman et al, 2001 \\
\hline
\end{tabular}

Evidence to suggest a possible association or risk with cannabis use

\begin{tabular}{|c|c|c|}
\hline Schizophrenia & Schizophrenia is associated with cannabis use but no causal role of cannabis has been found. & $\begin{array}{l}\text { Semple et al, } 2005 \\
\text { Marconi et al, } 2016 \\
\text { Aas et al, } 2018 \\
\text { Gage et al, } 2013\end{array}$ \\
\hline Depression & There is an unclear association between cannabis use and depression. & $\begin{array}{l}\text { Degenhardt et al, } 2003 \\
\text { Lev-Ran et al, } 2014\end{array}$ \\
\hline Testicular cancer & Possible increased risk of testicular cancer with heavy cannabis use. & $\begin{array}{l}\text { Gurney et al, } 2015 \\
\text { Callaghan } \text { et al, } 2017\end{array}$ \\
\hline $\begin{array}{l}\text { Cerebrovascular } \\
\text { events }\end{array}$ & Reports of cannabis use precipitating ischemic or transient cerebrovascular events. & $\begin{array}{l}\text { Wolff et al, } 2011 \text { \& } 2015 \\
\text { Thanvi et al, } 2009\end{array}$ \\
\hline Pregnancy & No clear evidence for direct harm of cannabis during pregnancy but no evidence that it is safe. & El Marroun et al, 2018 \\
\hline Youth & $\begin{array}{l}\text { Some evidence for a negative impact on brain development when cannabis is used in adolescents } \\
\text { and youth. }\end{array}$ & $\begin{array}{l}\text { Meier et al, } 2012 \\
\text { Brumback et al, } 2016\end{array}$ \\
\hline \multicolumn{3}{|c|}{ Evidence does not support an association with cannabis use } \\
\hline Lung Cancer & No independent association of cannabis use with lung cancer when tobacco use is controlled for. & Zhang et al, 2015 \\
\hline Cancer (others) & No clear association of cannabis use with any other cancers known to be related to tobacco use. & Sidney et al, 1997 \\
\hline Cardiovascular & No evidence for long-term changers in cardiovascular-related mortality with cannabis use. & Frost et al, 2013 \\
\hline
\end{tabular}

Abbreviation: COPD, chronic obstructive pulmonary disease 
Acute cannabis exposure also results in marked disturbances in cognition, perception, behaviour and level of consciousness. It is generally well accepted that cannabis impairs memory and concentration, although with the legalization of cannabis in Canada it has become more relevant to formally characterize the acute effects on cognition and the implications thereof. In a study examining patients using cannabis for pain, in addition to psychotic symptoms and adverse mental health outcomes, short-term cognitive impairment and increased motor-vehicle accidents (MVA) were reported [67]. Results of reports on the impact of cannabis use and risk of MVAs are conflicting; however, a meta-analysis conducted in 2012, reported an overall significant risk of MVAs with cannabis use [68] and THC was found to impair performance in driving simulation [69]. As a possible contributor for the negative impact on driving, cannabis use has been found to impair visuomotor skills, oculomotor function and motor learning both during acute exposure and has long-lasting effects in frequent users [70]. Increased numbers of MVAs, including fatal MVAs, are reported in the media to be associated with cannabis legalization. Additionally, fatal MVAs have been identified in Canada as a domain of cannabis-attributable mortality [71]. The most robust impairments associated with acute cannabis exposure affect memory. In a placebo-controlled trial in nondependent cannabis smokers, acute cannabis was found to impair verbal recall and working memory [72]. Other studies support these findings with further evidence for significant impairments in working memory, immediate and delayed recall and failure to use semantic processing and organization to optimize memory encoding and retrieval [69]. Overall, acute exposure to cannabis produces significant cognitive impairment in users and can result in a variety of other psychiatric symptoms ranging from mild and short-lasting to severe and prolonged.

\section{Impact of chronic consumption}

In addition to short-term effects on cognition, regular cannabis use has been associated with long-term deficits in cognitive performance including memory, attention, verbal learning and decline in IQ that may not be reversible after cessation of cannabis use $[3,73,74]$. These deficits exist during cannabis exposure and do not to return to baseline between cannabis uses. The long-lasting adaptations are hypothesized to be due to permanent adaptations in brain circuitry, including the endogenous cannabinoid system, as well as to irreversible hippocampal volumetric alteration $[75,76]$. The long-term impacts of cannabis use on cognition are associated with duration, frequency, estimated cumulative dose and age at initiation of use. Both the age at initiation of use and the frequency of use are also associated with lower SES and other negative psychosocial outcomes such as lower college degree completion and use of other drugs [3]. In order to assess whether SES could explain the association between cannabis use and cognition, a recent study reported that even after controlling for SES, significant reductions in IQ were observed in separated cohorts of middle and highSES groups [77]. The effects on memory are thought to persist up to one month after cessation of cannabis use and may persist even longer for those who were adolescents at initiation of use. Further studies are required to understand the long-term impact of cannabis use on cognitive function.

Long-term use of cannabis has been extensively associated with a variety of mental health conditions, and an understanding of these associations is critical for both medicinal and recreational users of cannabis. Here we will discuss the evidence for the association of cannabis with psychosis, schizophrenia, anxiety and depression. In addition to these disorders, patients diagnosed with cannabis use disorder (dependence or addiction) have a higher risk of developing other substance-use disorders, eating disorders, conduct disorders and personality disorders $[3,60]$.

\section{Association with psychosis}

Cannabis-induced psychosis is a rare acute phenomenon that most commonly occurs in frequent users of cannabis as opposed to intermittent users. It is characterized by a sudden onset of emotional lability and paranoid symptoms beginning 24 hours to one week after cannabis use. It is also referred to as acute psychosis with cannabis use and is grouped into the DSM-5 categorization of substanceinduced psychotic disorders. A distinction between CIP and early schizophrenic symptoms has been controversial in the past, and several studies have tried to distinguish the two. Evidence now supports a clear difference between CIP and subsequent risk for psychiatric disorders and from other related psychoses, even though the two are still intricately associated [78]. Cannabis-induced psychosis can resolve after onset; however, it persists in a subset of individuals, particularly those who continue to use cannabis or relapse [66]. In addition to acute CIP or cannabis-related psychoses, separate psychiatric disorders can develop later in life in frequent cannabis users. Previous CIP is a significant risk factor for the development of psychiatric disorders later in life. Of those who initially present with non-affective psychosis, $50 \%$ go on to develop an independent psychiatric disorder and only $7.7 \%$ who initially present with affective psychosis go on to develop an independent disorder[66]. Even without an episode of acute psychosis, chronic cannabis use is a risk factor for development of psychosis 
later in life and poses a significantly higher risk for individuals with other independent risk factors for psychiatric illness including family history [79].

\section{Association with schizophrenia}

Among the associations of psychiatric disease and cannabis use, schizophrenia is by far the most widely reported. Schizophrenia is a mental and behavioral disorder characterized by distortions in thinking, perception, emotions, language, sense of self and behavior [3]. Cannabis use has been identified as a risk factor for the development of schizophrenia $[80,81]$. However, regular cannabis use is also significantly more common among individuals with schizophrenia. Several possible explanations for this association between cannabis use and schizophrenia have been proposed. Whether cannabis use can be described as an overall risk factor for the development of schizophrenia is still unclear; however, it is likely to be a risk in certain circumstances, including use at a young age and with frequent and high consumption of cannabis. Use of high doses of cannabis are more strongly associated with risk of schizophrenia with an odds ratio of 3.9 in the heaviest cannabis users [82]. Use of cannabis before 18 years of age is also associated with a 2.3 times greater risk of receiving a diagnosis of schizophrenia, and in females use before 16 years of age is also associated with schizotypy symptoms $[3,83,84]$. Other studies report that there were pre-existing psychotic symptoms before regular cannabis use in individuals who go on to develop schizophrenia [83]. This suggests a possible reverse causation, where preschizophrenic individuals are more likely to use cannabis. Another study found that psychotic patients who used cannabis before illness onset had genetic risk factors suggesting their predisposition for psychiatric disease preceded or was a separate risk factor for cannabis use [85]. However, patients already at high risk, do appear to be more susceptible to the psychogenic effects of cannabis, and cannabis use in these individuals is associated with increases in positive and negative symptoms of psychosis [3]. The idea that common risk factors exist for cannabis use and the development of psychiatric disorders such as schizophrenia is referred to as the common cause hypothesis, a hypothesis that has been difficult to disprove due to confounding factors [3]. The lack of a clear increase in the incidence of schizophrenia, given the significant increase in cannabis use over the last several decades, is a piece of epidemiological data that supports a weak to non-existent causal role of cannabis for schizophrenia [86]. Overall there is a consensus that regular cannabis may pose a moderate increase in the risk for psychosis, but that this risk is much higher in individuals already at risk of psychiatric disease.

A recent systematic review of cannabis use in individuals with pre-existing psychotic disorders found that continued cannabis use is significantly associated with relapse, level of functioning and positive symptoms [87]. These findings are supported by considerable evidence that cannabis use is associated with worse outcomes in psychotic disorders [8890] and psychotic symptoms in schizophrenia [62]. Discontinuation of cannabis use should therefore be encouraged in those with existing psychosis or schizophrenia or with a new diagnosis of psychosis. There is clear evidence that schizophrenia and cannabis use are intertwined, and additional studies need to be done to elucidate whether a causal relationship exists between the two.

\section{Association with anxiety and depression}

Acutely, cannabis use can induce severe states of anxiety in individuals, which typically resolve within a maximum of 12 hours. Evidence also supports an increased risk for elevated anxiety symptoms in regular cannabis users. A meta-analysis of 40 studies revealed a 1.15 increased risk of developing elevated anxiety symptoms [91]. On the other hand, individuals with social anxiety disorder are more susceptible to cannabis-related impairment and have an increased risk of developing cannabis use disorder [92,93]. Another study found a possible reverse causation where generalised anxiety symptoms were associated with both an increase in likelihood to initiate cannabis use and an increased frequency of cannabis use [94]. The evidence supporting an increased risk for adverse outcomes following cannabis use in individuals with pre-existing anxiety is much better characterized [95]. Thus, individuals with anxiety should be considered at an increased risk of developing cannabis use disorder. Collectively, and as seen in other sections of this report, the relationship between anxiety and cannabis use and potential risk to users is difficult to discern due to the difficulty in separating confounding factors and in proving a causal role.

Rates of depressive disorders are also higher in cannabis users; however, only modest associations have been found in meta-analyses and evidence to support a causal relationship is minimal and in need of further longitudinal evaluation [3]. A literature review conducted in 2003 confirmed an association between heavy cannabis use and depressive symptoms; however, researchers could neither confirm a causal relationship nor control for common causes [96]. A more recent meta-analysis of the literature on cannabis use and depression came to the same conclusions, reflecting the continued need for studies examining the risks of cannabis 
use [97]. Cannabis use has also been linked to an increase in suicidal ideation and/or attempts [98,99], particularly with use during adolescence and more commonly in females [100]. However, a longitudinal study conducted in 2009 found no increase in suicide completion in cannabis users [101], and an additional meta-analysis of the literature conducted in 2016 reported no clear evidence to support cannabis use as a risk factor for suicidality [102]. In addition, cannabis users with pre-existing depression may experience increased suicidal ideation [103], although conflicting findings showing no increase in suicidal ideation have also been reported [104]. Similar to the findings in individuals with pre-existing anxiety, cannabis use in patients with preexisting depression is associated with worse recovery of symptoms and outcomes [105].

Interestingly, despite reported associations of cannabis use and worsening anxiety and depressive symptoms, medicinal cannabis is used as a treatment for both anxiety and depression. Due to the potential exacerbation of these conditions with cannabis, patients who are using this as a treatment should be monitored closely, and more evidence is required before cannabis can be accepted as an evidencebased treatment. In addition, medical professionals should be sure to review the literature and evidence for prescribing of medical cannabis, which is not covered in this review.

\section{Association with drug addiction}

Historically the addiction liability of cannabis has been controversial; however, evidence clearly indicates that chronic cannabis use can lead to addiction and dependence (both components of cannabis use disorder) $[106,107]$. In a recent longitudinal cohort study, cannabis dependence was found to have a comparable impact to alcohol dependence on socioeconomic concerns [108]. According to the diagnostic criteria for cannabis use disorder, approximately $9 \%$ of those exposed to cannabis will become addicted [109]. Cannabis use disorder is characterized by behavioural, cognitive and physiological phenomena and in North America is diagnosed based on DSM-5 criteria for the presence of at least two of the following:

1) use in larger amounts or for longer than indicated;

2) unsuccessful efforts to cut down use;

3) great deal of time spent to obtain, use and recover from the substance;

4) craving or strong desire to use the substance;

5) recurrent use results in failure to fulfil major obligations;

6) continued use despite recurrent social or interpersonal problems;

7) important activities reduced because of use;

8) recurrent use in physically hazardous situations;
9) continued use despite knowledge of problems caused by use of the substance;

10) evidence of tolerance; and

11) physiological withdrawal upon cessation of use.

While many factors, including genetics and SES, contribute to risk of developing cannabis use disorder, the risk is much higher in younger age groups and the risk of developing new onset dependence after 25 years of age is much lower [110]. Another study estimated that approximately one in ten people who have ever used cannabis will become dependent and that a higher risk is associated with more frequent use [107]. Treatment for cannabis use disorder encompasses acute management of withdrawal (within one week after cessation of use) and longer-term management and support for addiction. Cannabis use disorder is managed similarly to other substance use disorders with the best evidence for a combination of cognitive behavioural therapy, motivational enhancement therapy, and contingency management to achieve the best abstinence outcomes [111]. Due to the misguided perceptions around the addiction liability of cannabis, increased education about the prevalence and risk of cannabis use disorder is necessary to ensure individuals are best prepared to safely use cannabis with its recent legalization in Canada.

\section{Deaths related to cannabis use}

While excessive ingestion of cannabis can lead to toxicity and produce some of the adverse effects discussed in this review, there is a not a direct risk of overdose causing death with excessive cannabis consumption. Cannabis is rarely considered to have a contributory or causal role in sudden unexpected death; as few as 35 case reports where cannabis use is hypothesized to directly contribute to death have been described despite widespread use [112]. In these cases, cardiovascular complications secondary to cannabis use are thought to have contributed to death [112]. Although it is rarely a direct cause of death, cannabis use does contribute to a significant number of deaths in both adults and children due to other circumstances. These include motor vehicle accidents, fires, drowning, negligence and violence leading to death of others, and are thought to be related to impaired mental status and judgement due to cannabis use.

In summary, the mental health consequences of cannabis use are still poorly understood and additional longitudinal studies are required to best inform the public on safe use. Cannabis use has clear associations with mental health conditions, including acute psychosis, schizophrenia and anxiety; however, it is rare that a direct causal role of cannabis use can be found due to the presence of 
confounding variables and common causes. The implications of cannabis legalization in Canada and potential changes in its patterns of use, as well as the increasing illegal use of cannabis worldwide, will have a profound impact on mental health and is a pressing concern for global health [113].

\section{Cannabis use during pregnancy and youth}

While cannabis use has been associated with negative outcomes in general populations, it is important to define any specific subpopulations that may be at an elevated risk for adverse events. In this section we will detail the evidence that exists for negative effects of cannabis use in selected subpopulations that are of societal concern.

\section{Cannabis use during pregnancy}

Cannabis is the most commonly used illicit substance during pregnancy [114]. Because of this, there is considerable concern regarding the impact of cannabis use during pregnancy on the developing fetus. This likely stems, in large part, from the known negative effects of the use of alcohol, tobacco and other illicit substances on fetal development. Thus, it is of clinical and societal importance to define the specific effects of cannabis use during pregnancy.

While there is an increasing amount of literature surrounding both the short- and long-term consequences of gestational cannabis exposure, much of the reported effects are inconsistent or of poor quality [115]. The most recent meta-analysis took data from 31 studies that assessed the effects of maternal cannabis use on adverse neonatal outcomes [115]. Based on pooled unadjusted data, cannabis use during pregnancy was associated with an increased risk of low birth weight and preterm delivery. Pooled data adjusted for tobacco use and other confounding factors showed no statistically significant increased risk for low birth weight. Thus, the available evidence does not identify a large negative effect of cannabis use during pregnancy on fetal development. It is important to note that these trials are not designed to prove that cannabis use is safe during pregnancy, only to define larger negative effects. Because of this, cannabis use cannot be declared safe and should still be cautioned during pregnancy.

\section{Cannabis use in youth}

The prolonged development of the human brain into at least the middle of the second decade of life provides the opportunity for psychoactive compounds to alter either the developmental trajectory or predispose individuals to the development of neurological or psychiatric conditions. Because cannabis is frequently experimented with during the teenage years, questions on whether this could impact neurological development are frequent, and a growing body of literature attempting to address this exists. The consensus emerging from these data demonstrates that regular, heavy cannabis use in youth is associated with a greater degree of negative outcomes than use during adulthood.

One longitudinal study of 113 young adults evaluated overall IQ, memory, processing speed, vocabulary, attention and abstract reasoning in both light and heavy users $>5$ joints per week) that were followed and had baseline testing prior to starting to use cannabis [116]. They demonstrated that current regular heavy users did significantly worse than non-users in overall IQ, processing speed, immediate and delayed memory. In contrast, the former cannabis users did not show any cognitive impairment. It was concluded that residual cannabis effects are evident beyond the acute intoxication period in current heavy users after taking into account pre-drug performance, but similar deficits were no longer apparent three months after cessation of regular use, even among former heavy using young adults.

A more recent and extensive study [73] analyzed a prospective birth cohort of 1,037 individuals followed from birth to 38 years of age (the Dunedin Study). Here, cannabis use information was collected in interviews at 18, 21, 26, 32 and 38 years of age and cognitive testing was conducted at ages 13 and 38 years of age. This revealed an association between cannabis use and broad, multi-domain neuropsychological decline after controlling for confounding factors. The highest risk groups were those that started use at a young age and heavy, persistent cannabis users. Unlike the above study, here they did not observe a reversal of neuropsychological deficits after cannabis abstinence, although a degree of reversal was noted.

In addition to changes on cognitive testing, studies employing brain imaging techniques have revealed structural changes associated with prolonged cannabis use and with initiation of use in youth. A recent review of this literature [117] demonstrated that consistent evidence exists for alterations in the medial temporal, frontal and cerebellar brain regions. Structural changes in both gray matter and white matter have been reported on functional MRI (fMRI) imaging. Within the gray matter, a reduction in hippocampal volume is one of the most consistently reported changes on brain imaging detected with cannabis use. Interestingly, an increased cerebellar volume and thickening within the prefrontal cortex was also found in cannabis users and was inversely correlated with age at onset of use. This was hypothesized to be due to a disruption of brain development (i.e., pruning) with cannabis use in adolescence. A variety of cross-sectional and longitudinal studies show evidence of 
deficits in white matter macrostructure in cannabis users, particularly with use during adolescence. Functional MRI data support neurocognitive deficits in cannabis users and found that earlier onset use and more intense use during adolescence are associated with more severe deficits. Consistently, structural changes identified in cannabis users appear to be more significant in individuals reporting use during adolescence and those who use large amounts of cannabis for a more prolonged period. Attempts to correlate these structural changes with the neuropsychological changes described has produced mixed results, likely owing to the low samples sizes and complex correlations attempted in the studies. Nonetheless, the existing evidence suggests cannabis use in youth and adolescence may have a negative impact on neurological development and functioning that persists later in life.

\section{Conclusions}

The recent changes to the regulation of cannabis use in Canada have sparked further discussion around the benefits and harms of cannabis use. The existing literature demonstrates that chronic cannabis use may lead to symptoms of COPD (chronic bronchitis), although there seems to be no evidence of associated alveolar damage. Data on cardiovascular risks with cannabis use are also variable, with no changes to overall mortality, although there is evidence to demonstrate that acute cannabis use increases risk of MI. Finally, the association between cancer and cannabis consumption, either harm or benefit, is lacking strong evidence due to the contamination of the data as a result of the smoking of tobacco products. The area of cannabis and cancer, therefore, needs dedicated research to help inform both patients and clinicians. Mental health is intricately associated with cannabis use. Many common causes that increase the risk of cannabis use are also associated with an independent increased risk of psychiatric disorders. Furthermore, reverse causation studies found an increased risk of cannabis use in several underlying psychiatric disorders. Thus, discerning a causative effect of cannabis for increased psychiatric disease is extremely difficult. Nonetheless, caution should be taken for cannabis use in those with pre-existing symptoms and additional risk factors for psychiatric disease as they are at an increased risk of experiencing adverse effects with cannabis, use including increased development of cannabis use disorder. As well, frequent and heavy cannabis use can increase the severity of several mental health disorders. Regarding specific subpopulations, the evidence does not identify direct harm to fetal development from gestational exposure to cannabis. In youth, however, cannabis use has been associated with a decrease in future intellectual capacity and this effect is increased with earlier exposure and heavy use.

Collectively, the scarcity of properly controlled clinical research investigating the impact of cannabis consumption on physical and mental health requires our attention. The scientific and medical communities need to band together and conduct the appropriate research to identify possible risks and benefits to various populations.

\section{Authors' contributions}

HLP, SKJ and CN wrote the manuscript and all authors read and approved the final manuscript.

\section{Funding}

This research was supported by Alberta Innovates - Health Solutions MD/PhD Studentships (HLP, SKJ and CN).

\section{References}

1. Pertwee RG. Cannabinoid pharmacology: The first 66 years. BrJ Pharmacol. 2009;147(S1):S163-S171. doi:10.1038/sj.bjp. 0706406

2. Zlas J, Stark H, Seligman J, et al. Early medical use of cannabis. Nature. 1993;363(6426):215-215. doi: $10.1038 / 363215 \mathrm{a} 0$

3. WHO. The health and social effects of nonmedical cannabis use. 2016. Available at: https://www.who.int/ substance_abuse/publications/cannabis_report/en

4. Hajizadeh M. Legalizing and regulating marijuana in Canada: Review of potential economic, social, and health impacts. Int J Health Policy Manag. 2016;5(8):453-456. doi:10.15171/ijhpm. 2016.63

5. Hathaway, AD. Review of [Dan Malleck. When good drugs go bad: Opium, medicine, and the origins of Canada's drug laws. 249 pp. Vancouver: UBC Press, 2015.] Sci Can. 2016;39(1), 118-119. doi:10.7202/1041390ar

6. Rotermann M, Langlois K. Prevalence and correlates of marijuana use in Canada, 2012. Health Rep. 2015;26(4):10-15. https://www150.statcan.gc.ca/n1/pub/82-003-x/2015004/ article/14158-eng.pdf. Accessed October 14, 2018.

7. Adler JN, Colbert JA. Medicinal use of marijuana: Polling results. N Engl J Med. 2013;368(22):e30. doi:10.1056/ NEJMclde1305159

8. Atakan Z. Cannabis, a complex plant: Different compounds and different effects on individuals. Ther Adv Psychopharmacol. 2012;2(6):241-254. doi:10.1177/2045125312457586

9. Mechoulam R, Parker LA. The endocannabinoid system and the brain. Annu Rev Psychol. 2013;64:21-47. doi:10.1146/ annurev-psych-113011-143739 
10. Rabe KF, Watz H. Chronic obstructive pulmonary disease. Lancet (London, England). 2017;389(10082):1931-1940. doi: 10.1016/S0140-6736(17)31222-9

11. Tashkin DP. Marijuana and lung disease. Chest. 2018;154(3): 653-663. doi:10.1016/J.CHEST.2018.05.005

12. Hancox RJ, Shin HH, Gray AR, Poulton R, Sears MR. Effects of quitting cannabis on respiratory symptoms. Eur Respir J. 2015;46(1):80-87. doi:10.1183/09031936.00228914

13. Tan WC, Lo C, Jong A, et al. Marijuana and chronic obstructive lung disease: a population-based study. CMAJ. 2009;180(8):814-820. doi:10.1503/cmaj.081040

14. Fligiel SEG, Roth MD, Kleerup EC, Barsky SH, Simmons MS, Tashkin DP. Tracheobronchial histopathology in habitual smokers of cocaine, marijuana, and/or tobacco*. Chest. 1997;112(2):319-326. doi:10.1378/CHEST.112.2.319

15. Roth MD, Arora A, Barsky SH, Kleerup EC, Simmons M, Tashkin DP. Airway inflammation in young marijuana and tobacco smokers. Am J Respir Crit Care Med. 1998;157(3): 928-937. doi:10.1164/ajrccm.157.3.9701026

16. Tashkin DP, Simmons MS, Sherrill DL, Coulson AH. Heavy habitual marijuana smoking does not cause an accelerated decline in FEV1 with age. Am J Respir Crit Care Med. 1997;155(1):141-148. doi:10.1164/ajrccm.155.1.9001303

17. Pletcher MJ, Vittinghoff E, Kalhan R, et al. Association between marijuana exposure and pulmonary function over 20 years. $J A M A$. 2012;307(2):173. doi:10.1001/jama.2011.1961

18. Sherrill DL, Krzyzanowski M, Bloom JW, Lebowitz MD. Respiratory effects of non-tobacco cigarettes: A longitudinal study in general population. Int J Epidemiol. 1991;20(1): 132-137. doi:10.1093/ije/20.1.132

19. Tashkin DP. Does marijuana pose risks for chronic airflow obstruction? Ann Am Thorac Soc. 2015;12(2):235-236. doi: 10.1513/AnnalsATS.201412-581ED

20. Tashkin DP, Coulson AH, Clark VA, et al. Respiratory symptoms and lung function in habitual heavy smokers of marijuana alone, smokers of marijuana and tobacco, smokers of tobacco alone, and nonsmokers. Am Rev Respir Dis. 1987;135(1):209-216. doi:10.1164/arrd.1987.135.1.209

21. Aldington S, Williams M, Nowitz M, et al. Effects of cannabis on pulmonary structure, function and symptoms. Thorax. 2007;62(12):1058-1063. doi:10.1136/thx.2006.077081

22. Tashkin DP, Calvarese BM, Simmons MS, Shapiro BJ. Respiratory status of seventy-four habitual marijuana smokers. Chest. 1980;78(5):699-706. doi:10.1378/chest.78.5.699. Accessed November 3, 2018.

23. Hancox RJ, Poulton R, Ely M, et al. Effects of cannabis on lung function: A population-based cohort study. Eur Respir J. 2010;35(1):42-47. doi:10.1183/09031936.00065009

24. Morris MA, Jacobson SR, Kinney GL, et al. Marijuana use associations with pulmonary symptoms and function in tobacco smokers enrolled in the Subpopulations and Intermediate Outcome Measures in COPD Study (SPIROMICS). Chronic Obstr Pulm Dis. 2018;5(1):46-56. doi: 10.15326/jcopdf.5.1.2017.0141

25. Woodruff PG, Koth LL, Yang YH, et al. A distinctive alveolar macrophage activation state induced by cigarette smoking. Am J Respir Crit Care Med. 2005;172(11):1383-1392. doi:10.1164/ rccm.200505-686OC

26. Barbers RG, Gong H, Tashkin DP, Oishi J, Wallace JM. Differential examination of bronchoalveolar lavage cells in tobacco cigarette and marijuana smokers. Am Rev Respir Dis. 1987;135(6):1271-1275. doi:10.1164/arrd.1987.135.6.1271. Accessed November 3, 2018.

27. Baldwin GC, Tashkin DP, Buckley DM, Park AN, Dubinett SM, Roth MD. Marijuana and cocaine impair alveolar macrophage function and cytokine production. Am J Respir Crit Care Med. 1997;156(5):1606-1613. doi:10.1164/ajrccm. 156.5.9704146

28. Shay AH, Choi R, Whittaker K, et al. Impairment of antimicrobial activity and nitric oxide production in alveolar macrophages from smokers of marijuana and cocaine. J Infect Dis. 2003;187(4):700-704. doi:10.1086/368370

29. Kagen SL, Kurup VP, Sohnle PG, Fink JN. Marijuana smoking and fungal sensitization. J Allergy Clin Immunol. 1983;71(4):389-393. doi:10.1016/0091-6749(83)90067-2 . Accessed November 3, 2018.

30. Kalant H. Medicinal use of cannabis: History and current status. Pain Res Manag. 2001;6(2):80-91. doi: 10.1155/2001/469629. Accessed April 7, 2019.

31. Sharkey KA, Darmani NA, Parker LA. Regulation of nausea and vomiting by cannabinoids and the endocannabinoid system. Eur J Pharmacol. 2014;722:134-146. doi:10.1016/ j.ejphar.2013.09.068

32. Allen JH, de Moore GM, Heddle R, Twartz JC. Cannabinoid hyperemesis: Cyclical hyperemesis in association with chronic cannabis abuse. Gut. 2004;53(11):1566-1570. doi:10.1136/gut. 2003.036350

33. Goyal H, Singla U, Gupta U, May E. Role of cannabis in digestive disorders. Eur J Gastroenterol Hepatol. 2017;29(2): 135-143. doi:10.1097/MEG.0000000000000779

34. Boles RG, Chun N, Senadheera D, et al. Cyclic vomiting syndrome and mitochondrial DNA mutations. Lancet. 1997;350(9087):1299-300. doi:10.1016/ S0140-6736(05)62477-4

35. Richards JR. Cannabinoid hyperemesis syndrome: Pathophysiology and treatment in the emergency department. J Emerg Med. 2018;54(3):354-363. doi:10.1016/j.jemermed. 2017.12.010 
36. Goyal H, Awad HH, Ghali JK. Role of cannabis in cardiovascular disorders. J Thorac Dis. 2017;9(7):2079-2092. doi:10.21037/jtd.2017.06.104

37. Howlett AC, Barth F, Bonner TI, et al. International Union of Pharmacology. XXVII. Classification of cannabinoid receptors. Pharmacol Rev. 2002;54(2):161-202. doi:10.1124/pr. 54.2.161. Accessed November 4, 2018.

38. Hiley CR, Ford WR. Cannabinoid pharmacology in the cardiovascular system: Potential protective mechanisms through lipid signalling. Biol Rev Camb Philos Soc. 2004;79(1): 187-205. doi: 10.1017/s1464793103006201. Accessed November 4, 2018.

39. Steffens S, Pacher P. Targeting cannabinoid receptor CB2 in cardiovascular disorders: Promises and controversies. $\mathrm{BrJ}$ Pharmacol. 2012;167(2):313-323. doi:10.1111/j. 1476-5381.2012.02042.x

40. Sidney S. Cardiovascular consequences of marijuana use. J Clin Pharmacol. 2002;42(S1):64S-70S. doi:10.1002/j. 1552-4604.2002.tb06005.x. Accessed November 4, 2018.

41. Jones RT. Cardiovascular system effects of marijuana. J Clin Pharmacol. 2002;42(S1):58S-63S. doi:10.1002/j. 1552-4604.2002.tb06004.x. Accessed November 4, 2018.

42. Korantzopoulos P, Liu T, Papaioannides D, Li G, Goudevenos JA. Atrial fibrillation and marijuana smoking. Int J Clin Pract. 2007;62(2):308-313. doi:10.1111/j.1742-1241.2007.01505.x

43. Casier I, Vanduynhoven P, Haine S, Vrints C, Jorens PG. Is recent cannabis use associated with acute coronary syndromes? An illustrative case series. Acta Cardiol. 2014;69(2): 131-136. doi:10.2143/AC.69.2.3017293

44. Deharo P, Massoure P-L, Fourcade L. Exercise-induced acute coronary syndrome in a 24 -year-old man with massive cannabis consumption. Acta Cardiol. 2013;68(4):425-428. doi: 10.2143/AC.68.4.2988898

45. Mittleman MA, Lewis RA, Maclure M, Sherwood JB, Muller JE. Triggering myocardial infarction by marijuana. Circulation. 2001;103(23):2805-2809. doi:10.1161/01.cir.103.23.2805. Accessed November 4, 2018.

46. Frost L, Mostofsky E, Rosenbloom JI, Mukamal KJ, Mittleman MA. Marijuana use and long-term mortality among survivors of acute myocardial infarction. Am Heart J. 2013;165(2):170-175. doi:10.1016/j.ahj.2012.11.007

47. Wolff V, Schlagowski A-I, Rouyer O, et al.

Tetrahydrocannabinol induces brain mitochondrial respiratory chain dysfunction and increases oxidative stress: A potential mechanism involved in cannabis-related stroke. Biomed Res Int. 2015;2015:323706. doi:10.1155/2015/323706

48. Wolff V, Lauer V, Rouyer O, et al. Cannabis use, ischemic stroke, and multifocal intracranial vasoconstriction. Stroke. 2011;42(6):1778-1780. doi:10.1161/STROKEAHA. 110.610915
49. Thanvi BR, Treadwell SD. Cannabis and stroke: is there a link? Postgrad Med J. 2009;85(1000):80-83. doi:10.1136/pgmj. 2008.070425

50. Marselos M, Karamanakos P. Mutagenicity, developmental toxicity and carcinogenicity of cannabis. Addict Biol. 1999;4(1): 5-12. doi:10.1080/13556219971786

51. Novotný M, Merli F, Wiesler D, Fencl M, Saeed T. Fractionation and capillary gas chromatographic-mass spectrometric characterization of the neutral components in marijuana and tobacco smoke condensates. J Chromatogr $A$. 1982;238(1):141-150. doi:10.1016/S0021-9673(00)82720-X

52. Bhattacharyya S, Mandal S, Banerjee S, Mandal GK, Bhowmick AK, Murmu N. Cannabis smoke can be a major risk factor for early-age laryngeal cancer-a molecular signaling-based approach. Tumor Biol. 2015;36(8):6029-6036. doi:10.1007/s13277-015-3279-4

53. Callaghan RC, Allebeck P, Sidorchuk A. Marijuana use and risk of lung cancer: A 40-year cohort study. Cancer Causes Control. 2013;24(10):1811-1820. doi:10.1007/s10552-013-0259-0

54. Berthiller J, Straif K, Boniol M, et al. Cannabis smoking and risk of lung cancer in men: A pooled analysis of three studies in Maghreb. J Thorac Oncol. 2008;3(12):1398-1403. doi:10.1097/ JTO.0B013E31818DDCDE

55. Zhang LR, Morgenstern H, Greenland S, et al. Cannabis smoking and lung cancer risk: Pooled analysis in the International Lung Cancer Consortium. Int J Cancer. 2015;136(4):894-903. doi:10.1002/ijc.29036

56. Velasco G, Sánchez C, Guzmán M. Towards the use of cannabinoids as antitumour agents. Nat Rev Cancer. 2012;12(6): 436-444. doi:10.1038/nrc3247

57. Sidney S, Quesenberry CP, Friedman GD, Tekawa IS. Marijuana use and cancer incidence (California, United States). Cancer Causes Control. 1997;8(5):722-728. Available at: https:// www.jstor.org/stable/3552780. Accessed November 5, 2018.

58. Gurney J, Shaw C, Stanley J, Signal V, Sarfati D. Cannabis exposure and risk of testicular cancer: A systematic review and meta-analysis. BMC Cancer. 2015;15(1):897. doi:10.1186/ s12885-015-1905-6

59. Callaghan RC, Allebeck P, Akre O, McGlynn KA, Sidorchuk A. Cannabis use and incidence of testicular cancer: A 42-year follow-up of Swedish men between 1970 and 2011. Cancer Epidemiol Biomarkers Prev. 2017;26(11):1644-1652. doi: 10.1158/1055-9965.EPI-17-0428

60. Guttmannova K, Kosterman R, White HR, et al. The association between regular marijuana use and adult mental health outcomes. Drug Alcohol Depend. 2017;179:109-116. doi: 10.1016/j.drugalcdep.2017.06.016

61. Lisdahl KM, Wright NE, Medina-Kirchner C, Maple KE, Shollenbarger S. Considering cannabis: The effects of regular cannabis use on neurocognition in adolescents and young 
adults. Curr Addict Reports. 2014;1(2):144-156. doi:10.1007/ s40429-014-0019-6

62. Foti DJ, Kotov R, Guey LT, Bromet EJ. Cannabis use and the course of schizophrenia: 10-year follow-up after first hospitalization. Am J Psychiatry. 2010;167(8):987-993. doi: 10.1176/appi.ajp.2010.09020189

63. Gibbs M, Winsper C, Marwaha S, Gilbert E, Broome M, Singh SP. Cannabis use and mania symptoms: A systematic review and meta-analysis. J Affect Disord. 2015;171:39-47. doi: 10.1016/j.jad.2014.09.016

64. Grotenhermen F. Pharmacokinetics and pharmacodynamics of cannabinoids. Clin Pharmacokinet. 2003;42(4):327-360. doi: 10.2165/00003088-200342040-00003

65. Ashton $\mathrm{CH}$. Pharmacology and effects of cannabis: a brief review. Br J Psychiatry. 2001;178:101-106. doi: 10.1192/bjp. 178.2.101. Accessed November 10, 2018.

66. Shah D, Chand P, Bandawar M, Benegal V, Murthy P. Cannabis induced psychosis and subsequent psychiatric disorders. Asian J Psycbiatr. 2017;30:180-184. doi:10.1016/j.ajp. 2017.10.003

67. Nugent SM, Morasco BJ, O'Neil ME, et al. The effects of cannabis among adults with chronic pain and an overview of general harms. Ann Intern Med. 2017;167(5):319. doi:10.7326/ M17-0155

68. Li M-C, Brady JE, DiMaggio CJ, Lusardi AR, Tzong KY, Li G. Marijuana use and motor vehicle crashes. Epidemiol Rev. 2012;34(1):65-72. doi:10.1093/epirev/mxr017

69. Lenné MG, Dietze PM, Triggs TJ, Walmsley S, Murphy B, Redman JR. The effects of cannabis and alcohol on simulated arterial driving: Influences of driving experience and task demand. Accid Anal Prev. 2010;42(3):859-866. doi:10.1016/ j.aap.2009.04.021

70. Huestegge L, Kunert H-J, Radach R. Long-term effects of cannabis on eye movement control in reading.

Psychopharmacology (Berl). 2010;209(1):77-84. doi:10.1007/ s00213-009-1769-z

71. Fischer B, Imtiaz S, Rudzinski K, Rehm J. Crude estimates of cannabis-attributable mortality and morbidity in Canadaimplications for public health focused intervention priorities. $J$ Public Health (Bangkok). 2016;38(1):183-188. doi:10.1093/ pubmed/fdv005

72. Hindocha C, Freeman TP, Xia JX, Shaban NDC, Curran HV. Acute memory and psychotomimetic effects of cannabis and tobacco both 'joint' and individually: A placebo-controlled trial. Psycbol Med. 2017;47(15):2708-2719. doi:10.1017/ S0033291717001222

73. Meier MH, Caspi A, Ambler A, et al. Persistent cannabis users show neuropsychological decline from childhood to midlife. Proc Natl Acad Sci. 2012;109(40):E2657-E2664. doi:10.1073/ pnas.1206820109
74. Auer R, Vittinghoff E, Yaffe K, et al. Association between lifetime marijuana use and cognitive function in middle age: The Coronary Artery Risk Development in Young Adults (CARDIA) Study. JAMA Intern Med. 2016;176(3):352-361. doi: 10.1001/jamainternmed.2015.7841

75. Solowij N, Pesa N. [Cognitive abnormalities and cannabis use]. Rev Bras Psiquiatr. 2010;32 Suppl 1:S31-40. http:// www.scielo.br/scielo.php? script $=$ sci_arttext\&pid $=$ S1516-44462010000500006\&lng $=$ en \&nrm=iso\&tlng=en. Accessed November 10, 2018.

76. Chye Y, Suo C, Yücel M, den Ouden L, Solowij N, Lorenzetti V. Cannabis-related hippocampal volumetric abnormalities specific to subregions in dependent users. Psychopharmacology (Berl). 2017;234(14):2149-2157. doi:10.1007/s00213-017-4620$\mathrm{y}$

77. Moffitt TE, Meier MH, Caspi A, Poulton R. Reply to Rogeberg and Daly: No evidence that socioeconomic status or personality differences confound the association between cannabis use and IQ decline. Proc Natl Acad Sci U S A. 2013;110(11):E980-2. doi:10.1073/PNAS.1300618110

78. Shrivastava A, Johnston M, Tsuang M. Cannabis use and cognitive dysfunction. Indian J Psycbiatry. 2011;53(3):187-191. doi:10.4103/0019-5545.86796

79. Kristensen K, Cadenhead KS. Cannabis abuse and risk for psychosis in a prodromal sample. Psychiatry Res. 2007;151(1-2): 151-154. doi:10.1016/j.psychres.2006.10.001

80. Andréasson S, Allebeck P, Engström A, Rydberg U. Cannabis and schizophrenia. A longitudinal study of Swedish conscripts. Lancet (London, England). 1987;2(8574):1483-1486. doi:10.1016/s0140-6736(87)92620-1. Accessed April 6, 2019.

81. Semple DM, McIntosh AM, Lawrie SM. Cannabis as a risk factor for psychosis: systematic review. J Psychopharmacol. 2005;19(2):187-194. doi:10.1177/0269881105049040

82. Marconi A, Di Forti M, Lewis CM, Murray RM, Vassos E. Meta-analysis of the association between the level of cannabis use and risk of psychosis. Schizophr Bull. 2016;42(5):1262-1269. doi:10.1093/schbul/sbw003

83. Hall W, Degenhardt L. Cannabis use and psychosis: a review of clinical and epidemiological evidence. Aust N Z J Psychiatry. 2000;34(1):26-34. doi:10.1046/j.1440-1614.2000.00685.x

84. Albertella L, Le Pelley ME, Copeland J. Cannabis use in early adolescence is associated with higher negative schizotypy in females. Eur Psychiatry. 2017;45:235-241. doi:10.1016/j.eurpsy. 2017.07.009

85. Aas M, Melle I, Bettella F, et al. Psychotic patients who used cannabis frequently before illness onset have higher genetic predisposition to schizophrenia than those who did not. Psychol Med. 2018;48(01):43-49. doi:10.1017/ S0033291717001209 
86. Gage SH, Zammit S, Hickman M. Stronger evidence is needed before accepting that cannabis plays an important role in the aetiology of schizophrenia in the population. F1000 Med Rep. 2013;5:2. doi:10.3410/M5-2

87. Schoeler T, Monk A, Sami MB, et al. Continued versus discontinued cannabis use in patients with psychosis: A systematic review and meta-analysis. The Lancet Psychiatry. 2016;3(3):215-225. doi:10.1016/S2215-0366(15)00363-6

88. Zammit S, Moore THM, Lingford-Hughes A, et al. Effects of cannabis use on outcomes of psychotic disorders: systematic review. Br J Psychiatry. 2008;193(05):357-363. doi:10.1192/ bjp.bp.107.046375

89. Seddon JL, Birchwood M, Copello A, et al. Cannabis use is associated with increased psychotic symptoms and poorer psychosocial functioning in first-episode psychosis: A report from the UK national EDEN study. Schizophr Bull. 2016;42(3): 619-625. doi:10.1093/schbul/sbv154

90. Faber G, Smid HGOM, Van Gool AR, Wunderink L, van den Bosch RJ, Wiersma D. Continued cannabis use and outcome in first-episode psychosis: Data from a randomized, openlabel, controlled trial. J Clin Psychiatry. 2012;73(5):632-638. doi: 10.4088/JCP.11m07069

91. Twomey CD. Association of cannabis use with the development of elevated anxiety symptoms in the general population: A meta-analysis. J Epidemiol Community Health. 2017;71(8):811-816. doi:10.1136/jech-2016-208145

92. Ecker AH, Buckner JD. Cannabis use behaviors and social anxiety: the roles of perceived descriptive and injunctive social norms. J Stud Alcohol Drugs. 2014;75(1):74-82. doi:10.15288/ jsad.2014.75.74. Accessed November 10, 2018.

93. Buckner JD, Schmidt NB, Lang AR, Small JW, Schlauch RC, Lewinsohn PM. Specificity of social anxiety disorder as a risk factor for alcohol and cannabis dependence. J Psychiatr Res. 2008;42(3):230-239. doi:10.1016/j.jpsychires.2007.01.002

94. Stapinski LA, Montgomery AA, Araya R. Anxiety, depression and risk of cannabis use: Examining the internalising pathway to use among Chilean adolescents. Drug Alcohol Depend. 2016;166:109-115. doi:10.1016/j.drugalcdep.2016.06.032

95. Buckner JD, Crosby RD, Wonderlich SA, Schmidt NB. Social anxiety and cannabis use: An analysis from ecological momentary assessment. J Anxiety Disord. 2012;26(2):297-304. doi:10.1016/j.janxdis.2011.12.006

96. Degenhardt L, Hall W, Lynskey M. Exploring the association between cannabis use and depression. Addiction. 2003;98(11): 1493-1504. doi:10.1046/j.1360-0443.2003.00437.x. Accessed November 10, 2018.

97. Lev-Ran S, Roerecke M, Le Foll B, George TP, McKenzie K, Rehm J. The association between cannabis use and depression: A systematic review and meta-analysis of longitudinal studies.
Psychol Med. 2014;44(04):797-810. doi:10.1017/

S0033291713001438

98. Silins E, Horwood LJ, Patton GC, et al. Young adult sequelae of adolescent cannabis use: an integrative analysis. The Lancet Psychiatry. 2014;1(4):286-293. doi:10.1016/ S2215-0366(14)70307-4

99. Serafini G, Pompili M, Innamorati M, Rihmer Z, Sher L, Girardi P. Can cannabis increase the suicide risk in psychosis? A critical review. Curr Pharm Des. 2012;18(32):5165-5187. doi: 10.2174/138161212802884663. Accessed April 6, 2019.

100. Halladay JE, Boyle MH, Munn C, Jack SM, Georgiades K. Sex differences in the association between cannabis use and suicidal ideation and attempts, depression, and psychological distress among Canadians. Can J Psychiatry. September 2018;64(5):345-350. doi:10.1177/0706743718804542

101. Price C, Hemmingsson T, Lewis G, Zammit S, Allebeck P. Cannabis and suicide: longitudinal study. BrJ Psychiatry. 2009;195(06):492-497. doi:10.1192/bjp.bp.109.065227

102. Borges G, Bagge CL, Orozco R. A literature review and metaanalyses of cannabis use and suicidality. J Affect Disord. 2016;195:63-74. doi:10.1016/j.jad.2016.02.007

103. Bahorik AL, Sterling SA, Campbell CI, Weisner C, Ramo D, Satre DD. Medical and non-medical marijuana use in depression: Longitudinal associations with suicidal ideation, everyday functioning, and psychiatry service utilization. I Affect Disord. 2018;241:8-14. doi:10.1016/j.jad.2018.05.065

104. Naji L, Rosic T, Dennis B, et al. The association between cannabis use and suicidal behavior in patients with psychiatric disorders: an analysis of sex differences. Biol Sex Differ. 2018;9(1):22. doi:10.1186/s13293-018-0182-x

105. Bahorik AL, Leibowitz A, Sterling SA, Travis A, Weisner C, Satre DD. Patterns of marijuana use among psychiatry patients with depression and its impact on recovery. J Affect Disord. 2017;213:168-171. doi:10.1016/j.jad.2017.02.016

106. Budney AJ, Roffman R, Stephens RS, Walker D. Marijuana dependence and its treatment. Addict Sci Clin Pract. 2007;4(1): 4-16. doi:10.1151/ascp07414. Accessed November 10, 2018.

107. Copeland J, Swift W. Cannabis use disorder: Epidemiology and management. Int Rev Psychiatry. 2009;21(2):96-103. doi: 10.1080/09540260902782745

108. Cerdá M, Moffitt TE, Meier MH, et al. Persistent cannabis dependence and alcohol dependence represent risks for midlife economic and social problems. Clin Psychol Si. 2016;4(6):1028-1046. doi:10.1177/2167702616630958

109. Lopez-Quintero C, Cobos JP de los, Hasin DS, et al. Probability and predictors of transition from first use to dependence on nicotine, alcohol, cannabis, and cocaine: Results of the National Epidemiologic Survey on Alcohol and Related Conditions (NESARC). Drug Alcohol Depend. 2011;115(1-2):120-130. doi:10.1016/j.drugalcdep.2010.11.004 
110. Winters KC, Lee C-YS. Likelihood of developing an alcohol and cannabis use disorder during youth: Association with recent use and age. Drug Alcohol Depend. 2008;92(1-3):239-247. doi:10.1016/j.drugalcdep.2007.08.005

111. Sherman BJ, McRae-Clark AL. Treatment of cannabis use disorder: Current science and future outlook. Pharmacotherapy. 2016;36(5):511-535. doi:10.1002/phar.1747

112. Drummer OH, Gerostamoulos D, Woodford NW. Cannabis as a cause of death: A review. Forensic Sci Int. 2019;298:298-306. doi:10.1016/j.forsciint.2019.03.007

113. Bloomfield MAP, Ashok AH, Volkow ND, Howes OD. The effects of $\Delta 9$-tetrahydrocannabinol on the dopamine system. Nature. 2016;539(7629):369-377. doi:10.1038/nature20153

114. Meyer JS, Piper BJ. Chapter 26. Developmental neurotoxicity of abused drugs. In: Gupta RC, ed. Reproductive and Developmental Toxicology. 1st ed. Academic Press; 2011. doi: 10.1016/b978-0-12-382032-7.10026-8.

115. El Marroun H, Brown QL, Lund IO, et al. An epidemiological, developmental and clinical overview of cannabis use during pregnancy. Prev Med (Baltim). 2018;116:1-5. doi:10.1016/j.ypmed.2018.08.036

116. Fried PA, Watkinson B, Gray R. Neurocognitive consequences of marihuana-A comparison with pre-drug performance. Neurotoxicol Teratol. 27(2):231-239. doi:10.1016/j.ntt. 2004.11.003

117. Brumback T, Castro N, Jacobus J, Tapert S. Effects of marijuana use on brain structure and function: Neuroimaging findings from a neurodevelopmental perspective. Int Rev Neurobiol. 2016;129:33-65. doi:10.1016/bs.irn.2016.06.004 